\title{
ORIGINAL
}

\section{GATA6, SF1, NGFIB and DAX1 in the remodeled subcapsular zones in primary aldosteronism}

\author{
Yasuhiro Nakamura, Yumi Kurotaki, Kazue Ise, Saulo J.A. Felizola, Keely M McNamara and \\ Hironobu Sasano
}

Department of Pathology, Tohoku University Graduate School of Medicine, Sendai 980- 8575, Japan

\begin{abstract}
The majority of the cases diagnosed as primary aldosteronism (PA) are caused by aldosterone-producing adenoma (APA) or idiopathic hyperaldosteronism (IHA). Histopathologically, both IHA and adjacent adrenal glands of APA demonstrate remodeled subcapsular zone (RSZ) but these zones in two disorders are markedly different in terms of

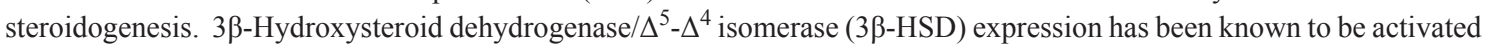
synergistically by GATA6 and SF1, and repressed by DAX1 through abolishing the activation. Nerve growth factorinduced clone B (NGFIB) is also known as one of the transcription factors to bind to and activate 3 $\beta$-HSD promoter. The results of our immunohistochemical analysis demonstrated the expression levels of 3 $\beta$-HSD in RSZ of IHA were higher than in RSZ of adjacent adrenals of APA, while those in the zona glomerulosa (ZG) of normal adrenal gland (NA) were in between these two RSZs. The expression levels of GATA6, SF1 and DAX1 did not prominently differ among these three types of adrenals, especially between in RSZs of IHA and APA cases, indicating the marked difference of 3 $\beta$-HSD expression was unlikely to be explained by the levels of these three factors. However, the levels of NGFIB expression were significantly higher in RSZ of IHA than in RSZ of adjacent adrenals of APA and the ZG of NA $(P<0.05)$, which may partly account for the expression levels of $3 \beta$-HSD among the three groups of adrenals. These results may imply NGFIB plays important roles in the marked differences in steroidogenic functions in the two distinct types of RSZ of PA cases.
\end{abstract}

Key words: Remodeled subcapsular zone, Primary aldosteronism, Immunohistochemistry

PRIMARY ALDOSTERONISM (PA) is one of the most common causes of secondary hypertension. Its underlying cause is usually classified into aldosterone-producing adenoma (APA) and idiopathic hyperaldosteronism (IHA) primarily based upon their histopathological findings. However, an evaluation of remodeling in the subcapsular zones has not necessarily been studied in detail in these disorders of PA. For instance, the great majority of studies have focused on adenoma itself rather than the adjacent non-neoplastic tissue, while in IHA tissue the specimens available for histopathological examination have become increasingly rare due to the predominate mode of treatment

Submitted Mar. 12, 2013; Accepted Jan. 17, 2014 as EJ13-0103 Released online in J-STAGE as advance publication Feb. 15, 2014

Correspondence to: Yasuhiro Nakamura, M.D., Ph.D., Department of Pathology, Tohoku University School of Medicine, 2-1 Seiryomachi, Aoba-ku, Sendai, 980- 8575 JAPAN.

E-mail: yasu-naka@patholo2.med.tohoku.ac.jp

(c) The Japan Endocrine Society being medical therapy alone rather than surgical intervention. In a few previous studies on histopathology of IHA cases, the remodeled subcapsular zones were reported to be associated with marked expression of steroidogenic enzymes including $3 \beta$-hydroxysteroid dehydrogenase $/ \Delta^{5}-\Delta^{4}$ isomerase (3ß-HSD), CYP11A1 and CYP21 $[1,2]$. The adrenal cortex adjacent to APA was also reported to be associated with histological findings similar to those of IHA but was not necessarily associated with abundance of steroidogenic enzymes as detected in IHA, with the possible exception of CYP21 $[1,2]$. Therefore, the expression patterns of these steroidogenic enzymes, especially $3 \beta-\mathrm{HSD}$, in the remodeled subcapsular zones of PA cases could be important differential markers between APA and IHA when surgical pathologists are requested to differentiate these disorders in the resected specimens of adrenal glands, although rare in the recent practice of surgical pathology. In addition, CYP11B2 expression as assessed by 
in situ hybridization and immunohistochemical analysis have been also reported in these remodeled subcapsular areas of these adrenals with PA [3, 4]. However, it is also true that the detailed characteristics of the remodeled subcapsular zones in both IHA and APA adjacent adrenal gland have remained largely unknown.

Martin et al. previously reported that the $3 \beta$-HSD type 2 (HSD3B2) promoter contains four consensus GATA regulatory motifs, an important target for GATA factors including GATA6 [5]. Functional effects of GATA6 on HSD3B2 have been previously demonstrated with coexpression of GATA6 and steroidogenic factor 1 (SF1), which did result in a synergistic activation of HSD3B2 promoter activity [5]. In addition, biological actions of these transcription factors are not necessarily confined to HSD3B2 activation because these factors were also reported to affect other steroidogenic factors such CYP11A1 and StAR [6-8]. The GATA6/SF1 signaling pathway above was also reported to be negatively regulated by DAX1 in their upstream because DAX1 was reported to oppose SF1-activated transcription and abolish the ability of GATA6/SF1 to increase their reporter activities of various types of steroidogenic enzymes in the adrenal gland $[6,9]$.

In addition to the GATA6/SF1/DAX1 pathway above, nerve growth factor induced-B (NGFIB) is also known as one of the transcription factors that bind to and activate HSD3B2, CYP21 and CYP11B2 promoters [10-12]. These important transcription factors have been all previously reported in human adrenal cortex but their possible involvement in the remodeled subcapsular zones of both IHA and APA adjacent adrenal gland has remained unknown [13-17]. Therefore, in this study, we immunolocalized GATA6, SF1, NGFIB and DAX1 in the remodeled sub-capsular zones of IHA and APA associated adrenal glands and compared these results with staining in the zona glomerulosa (ZG) of normal adrenal tissues in order to obtain the better understanding of these unique and interesting features of subcapsular zones of the adrenals associated with PA.

\section{Materials and methods}

\section{Human adrenal specimens}

Forty-nine human adrenal specimens were examined in this study. Twenty specimens of non-pathological adrenal glands (NA) from the patients with renal cell carcinoma, 18 cases of adjacent adrenal glands of APA and 11 cases of IHA were all retrieved from the surgical pathology files of Tohoku University Hospital, Sendai, Japan. All the specimens had been fixed in $10 \%$ formalin for $24-48 \mathrm{~h}$ at room temperature and embedded in paraffin wax. The research protocol for this study was approved by the Ethics Committee at Tohoku University School of Medicine.

\section{Antibodies}

Rabbit polyclonal antibodies for NGFIB, GATA6 and DAX1 were commercially obtained from Geneka Biotechnology (Montréal, Canada) and Santa Cruz Biotechnology, Inc. (Santa Cruz, CA, USA), respectively $[10,12,14-16,18,19]$. Mouse monoclonal antibody for SF1 was purchased from Perseus Proteomics Inc. (Tokyo, Japan). The polyclonal antibody for $3 \beta$-HSD was kindly provided by Dr. Mason (University of Edinburgh, Edinburgh, UK) [19]. This antibody recognized both type I and II isoforms of the enzyme. The polyclonal antibody for P450 17 $\alpha$ hydroxylase/17, 20-lyase (CYP17) was developed inhouse and its design and validation has been previously reported [20].

\section{Immunohistochemical staining}

Immunohistochemical analysis was performed employing the streptavidin-biotin amplification method using a Histofine Kit (Nichirei, Tokyo, Japan). Antigen retrieval was performed except for immunostaining of $3 \beta-H S D$ by heating the slides in an autoclave at $120^{\circ} \mathrm{C}$ for $5 \mathrm{~min}$ in citric acid buffer $(2 \mathrm{mM}$ citric acid and $9 \mathrm{mM}$ trisodium citrate dehydrate, $\mathrm{pH}$ 6.0) while for $3 \beta$-HSD no antigen retrieval was performed. The dilutions of the primary antibodies used in this study were: GATA6, 1:600; SF1, 1:100; NGFIB, 1:200; DAX1, 1:500, 3ß-HSD, 1:2,500; CYP17, 1:500, respectively. The antigen-antibody complex was visualized with 3,3'-diaminobenzidine solution [1 mM 3,3'diaminobenzidine, $50 \mathrm{mM}$ Tris- $\mathrm{HCl}$ buffer ( $\mathrm{pH}$ 7.6), and $0.006 \% \mathrm{H}_{2} \mathrm{O}_{2}$ ] and counterstained with hematoxylin. Normal rabbit IgG was also used instead of the primary antibodies as a negative control of immunstaining. In addition, preabsorption tests for CYP17, NGFIB and DAX1 were carried out by incubating the antibody-antigen mixture containing equal volumes of these optimally diluted antibodies and the corresponding peptides solution. The sequence of peptide for CYP17 was mixture of (i) LHHNEKEWHQPDQFC, (ii) KMNSDNGNAGPDQDSC and (iii) KKGKDFSG RPQMAC. The peptides for NGFIB and DAX1 were 
purchased from the companies above. No specific immunoreactivity was detected in these negative controls (data not shown).

\section{Evaluation of immunoreactivity}

In our present study, relative immunoreactivity in the subcapsular zones was evaluated by employing H-score, as described by McCarty et al. with some modifications [21]. Briefly, adrenocortical cells in the subcapsular zones were evaluated and $\mathrm{H}$-scores were subsequently generated by adding together $3 \times$ the percentage of strongly stained nuclei, $2 \times$ the percentage of moderately stained nuclei, $1 \times$ the percentage of weakly stained nuclei, and $0 \times$ the percentage of negative nuclei, yielding a possible range of 0-300. All the data obtained were presented as mean \pm S.E. Significant variation in H-score among all three groups above was assessed using one-way analysis of variance followed by TukeyKramer HSD multiple comparisons for post-hoc comparisons between individual groups. We also used a correlation coefficient $(r)$ and a regression equation in order to further examine the correlation of these two factors. $P<0.05$ was considered significant in this study.

\section{Results}

We defined the conventional ZG of NA and extension of the remodeled subcapsular zones in PA cases by careful histological examination, as well as $3 \beta-H S D$ and CYP17 immunoreactivities (Fig. 1-3). In the ZG of NA, 3 $\beta$-HSD immunoreactivity was pronouncedly detected but CYP17 immunoreactivity was completly absent (Fig. 1). GATA6, SF1, NGFIB and DAX1 immunoreactivities were all detected in the nuclei of cortical cells of the normal ZG (Fig. 1).

In IHA cases examined, the remodeled subcapsular zone was continuously detectable in the whole adrenal gland, associated with increased 3 $\beta$-HSD immunoreactivity compared to the ZG of NA (Fig. 2). GATA6, SF1, NGFIB and DAX1 nuclear immunoreactivities were all diffusely detectable in the nuclei of cortical cells of these subcapsular zones (Fig. 2).

In APA cases examined in this study, the remodeled subcapsular zone was also continuously detectable in the whole adrenal gland but associated with marked reduced immunoreactivity of $3 \beta$-HSD compared to the ZG of NA (Fig. 3). GATA6, SF1, NGFIB and DAX1 nuclear immunoreactivities were also detectable in the nuclei of cortical cells of the subcapsular zone in all the cases examiend (Fig. 3).

The level of GATA6 immunoreactivity was sinigificantly higher in the remodeled subcapsular zone of APA case than the ZG of NA $(P<0.05)$ (Fig. 4A). No significant difference of GATA6 expression level was detected between NA and IHA cases, or APA and IHA cases (Fig. 4A). The expression level of SF1 was significantly higher in the remodeled subcapsular zone of IHA than the ZG of NA $(P<0.05)$ (Fig. 4B). However, no significant difference was detected between NA and APA cases, or APA and IHA cases (Fig. 4B).

The level of NGFB1 immunoreactivity was significantly higher in the remodeled capsular zone of IHA cases than those of APA cases and the ZG of NA $(P<0.05)$ (Fig. 4C). The expression level of DAX1 was also significantly higher in the remodeled subcapsular zone of IHA and APA case than the ZG of NA $(P<0.05)$ (Fig. 4D) but not different between APA and IHA cases (Fig. 4D). The expression level of $3 \beta$-HSD was significantly higher in the remodeled capsular zone of IHA than those of APA cases and the ZG of NA $(P<0.05)$, and was also significantly lower in APA cases than the ZG of NA $(P<0.05)$ (Fig. 4E). The difference of correlation between relative expression levels of $3 \beta$-HSD and NGFIB in the ZG of NA and the remodeled subcapsular zones of IHA and adjacent adrenal gland to APA was summarized in Fig. 4F. The expression level of all these proteins examined was not significantly correlated with age or gender of the cases examined (data not shown).

\section{Discussion}

In our present study, we attempted to evaluate the underlying molecular or cellular basis of differences of steroidogenesis in the areas of non-neoplastic cortical remodeling reported APA and IHA. We examined four transcription factors from two different intracellular pathways previously reported to influence the transcription of steroidogenic enzymes in the adrenal cortex but have not been previously studied in PA. Of interest, among these four transcription factors examined, the increment of GATA6/SF1/DAX1 pathway was associated with subcapsular remodeling of both IHA and attached adrenals of APA but that of NGFIB was more pronounced in the former than the latter. These results did indicate that GATA6/SF1/DAX1 pathway was not, at least directly, involved in putative overproduction of the remodeled subcapsular zone in 

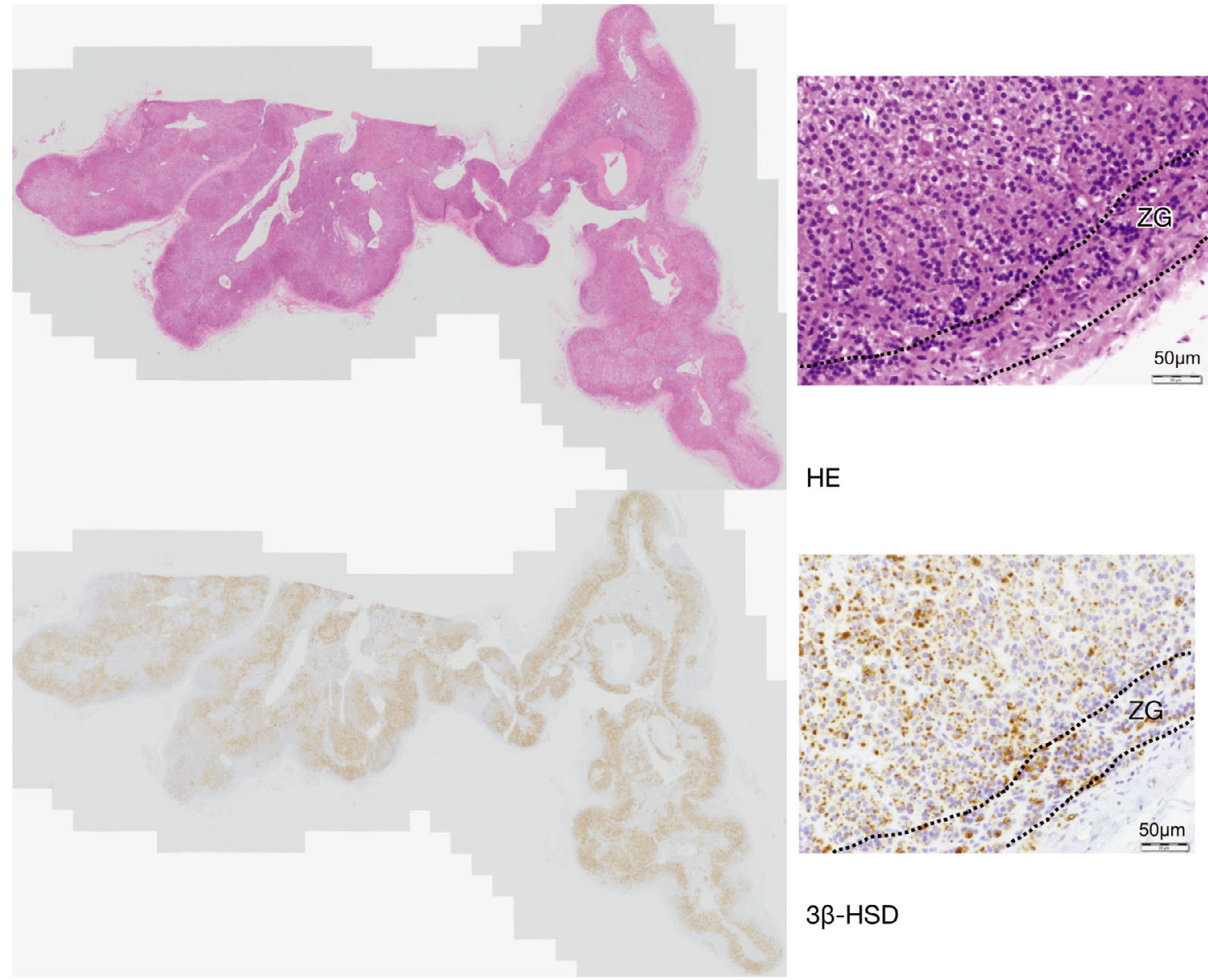

HE

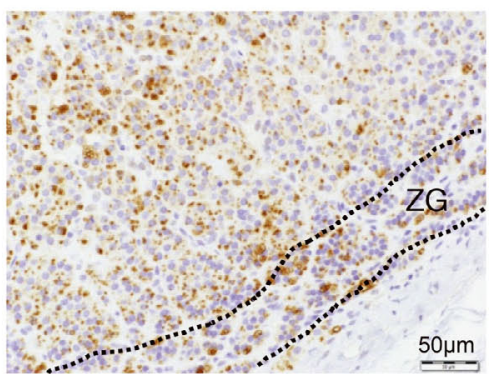

$3 \beta-H S D$

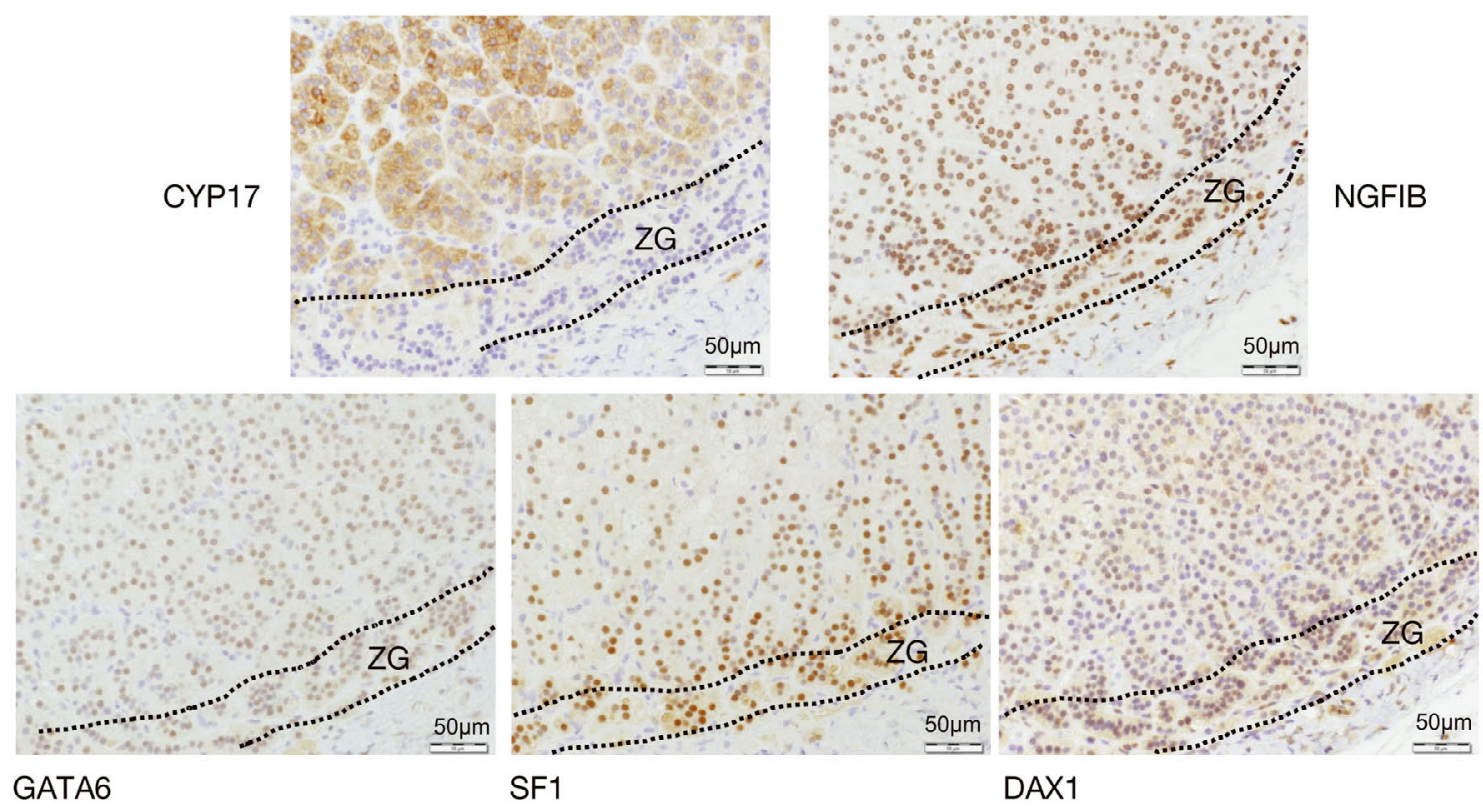

Fig. 1 Representative figures of the zona glomerulosa (ZG) of normal adrenal gland (NA). H\&E staining (HE) and immunohis-

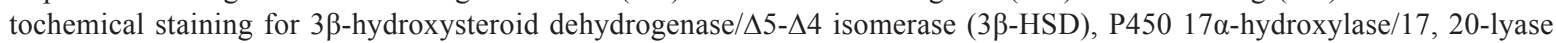
(CYP17), NGFIB, GATA6, SF1 and DAX1 were illustrated. H\&E stained images demonstrated the continuous ZG in the whole adrenal gland with immunopositivity of 3 $\beta$-HSD but not CYP17. GATA6, SF1, NGFIB, and DAX1 immunoreactivities were all detected in the nuclei of cortical cells of the normal ZG. 

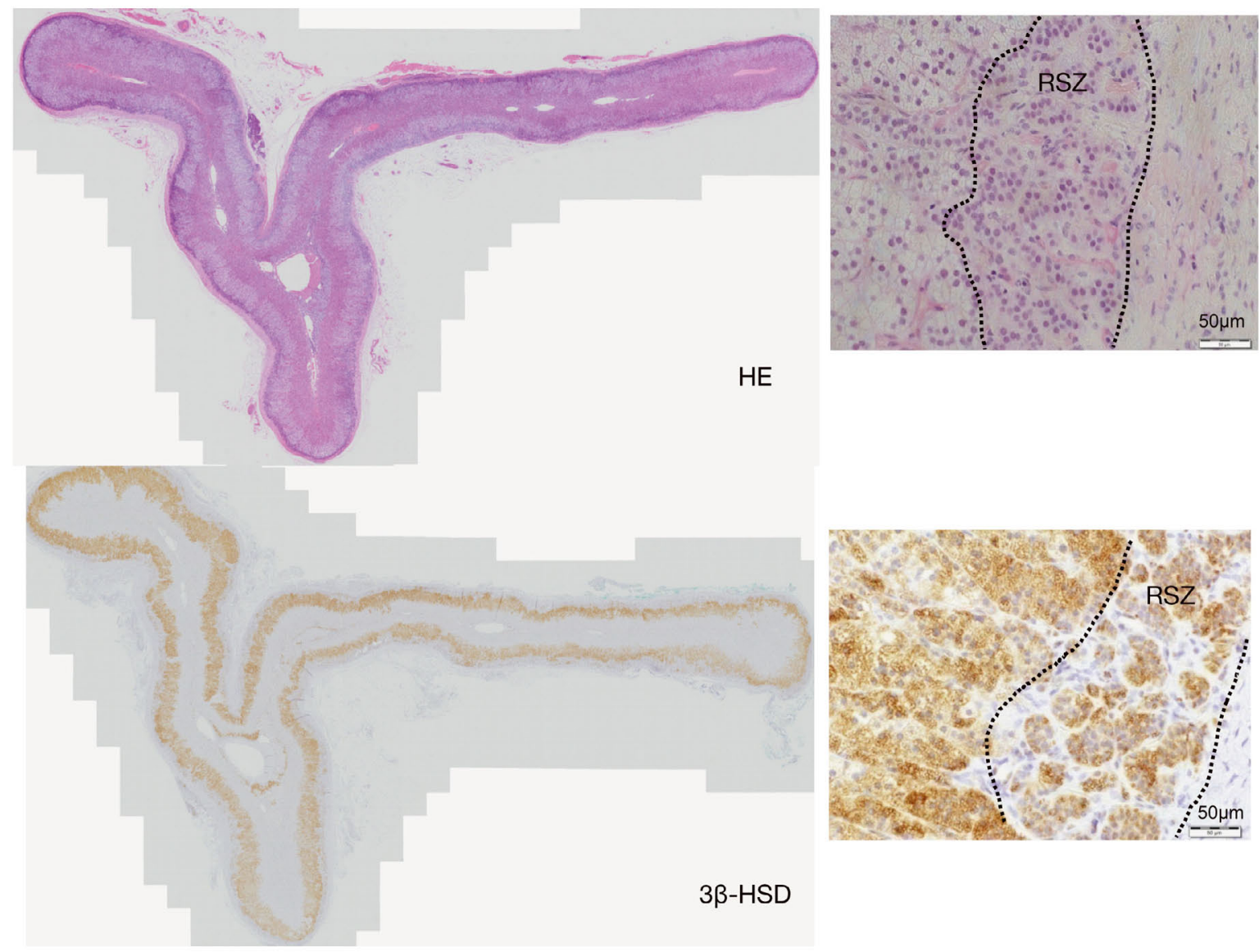

HE
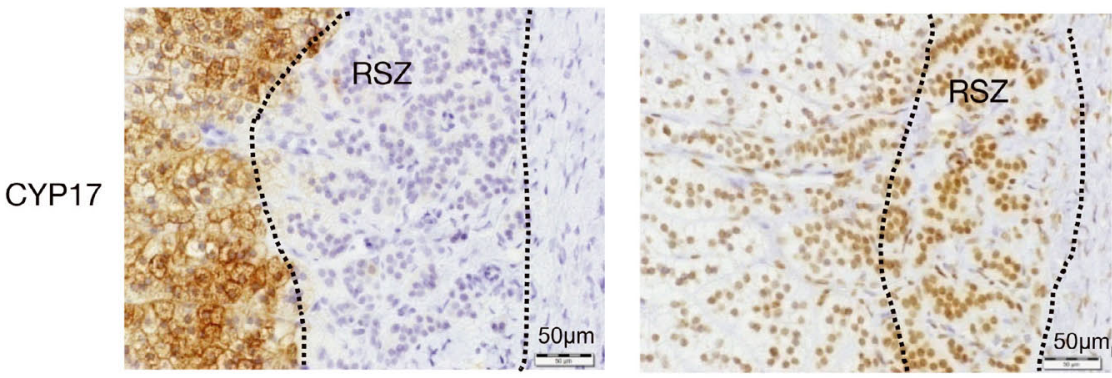

NGFIB
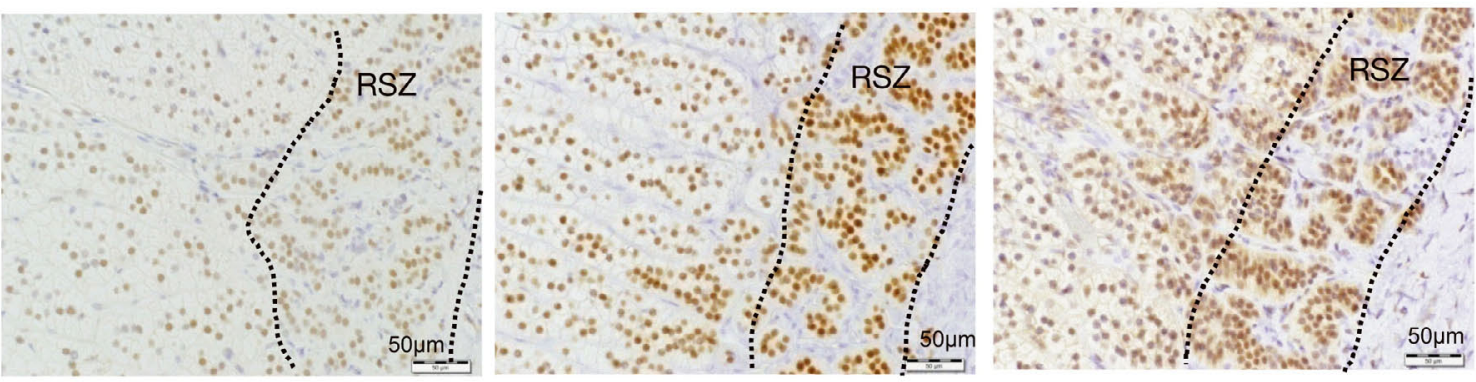

GATA6

SF1

DAX1

Fig. 2 Representative figures of the remodeled subcapsular zones (RSZ) of idiopathic hyperaldosteronism (IHA) demonstrated the continuous and RSZ in the whole adrenal gland with increased immunopositivity of $3 \beta$-hydroxysteroid dehydrogenase/ $\Delta 5-\Delta 4$

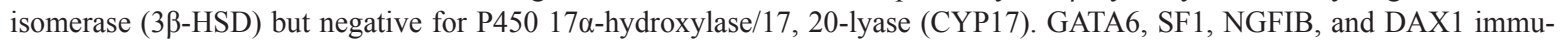
noreactivities were all detected in the nuclei of cortical cells of the RSZ. 

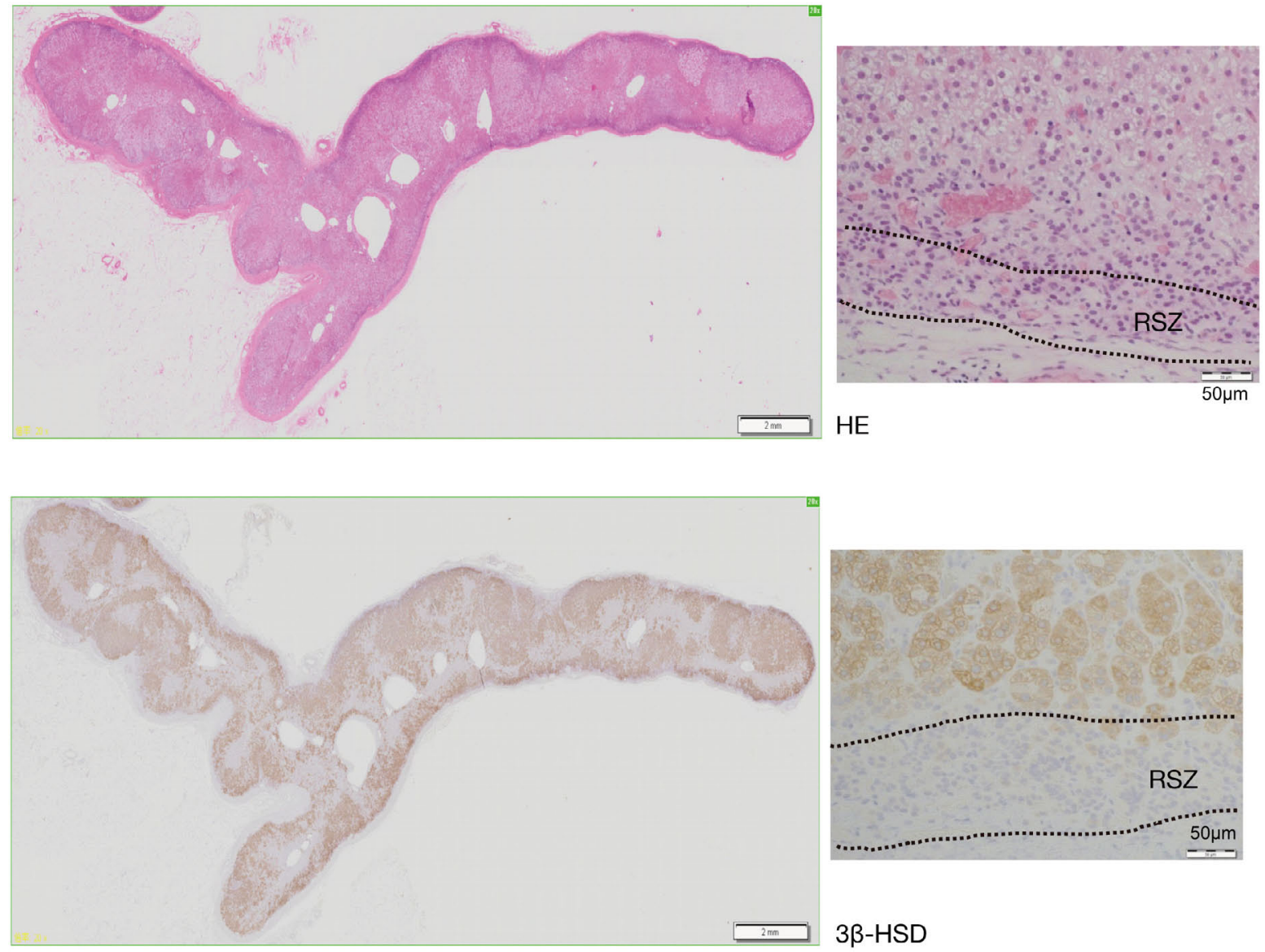

\section{3ß-HSD}
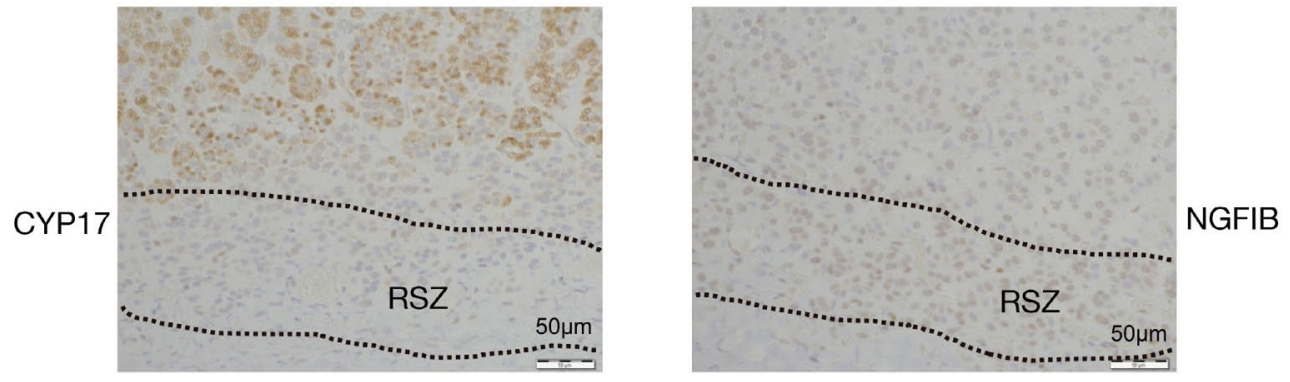

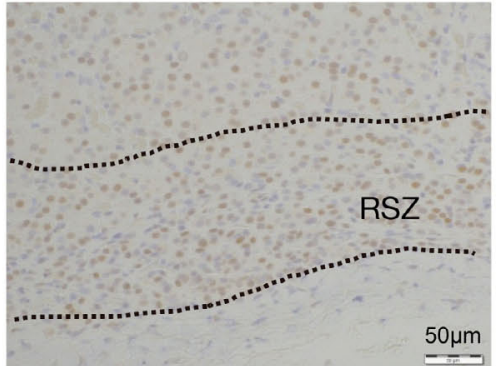

GATA6

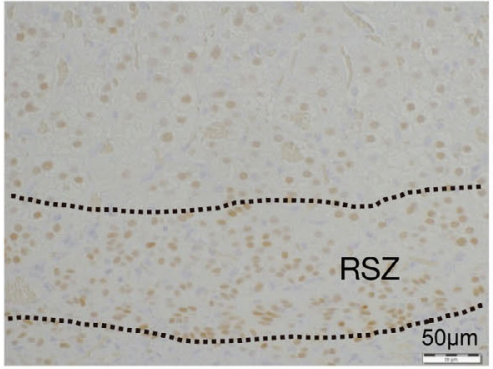

SF1

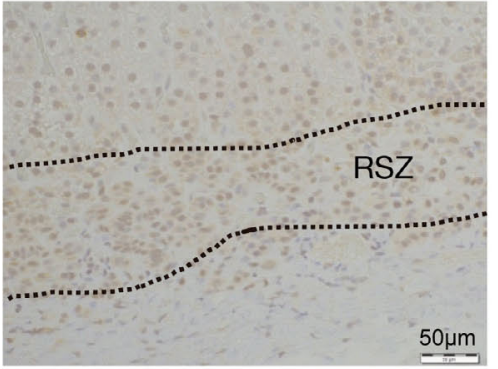

DAX1

Fig. 3 Representative figures of the remodeled subcapsular zones (RSZ) of adjacent adrenal gland in aldosterone-producing adenoma (APA) case showed the continuous RSZ in the whole adrenal gland with decreased immunopositivity of $3 \beta$-hydroxysteroid dehydrogenase/ $\Delta 5-\Delta 4$ isomerase (3 $\beta$-HSD) and negative for P450 17 $\alpha$-hydroxylase/17, 20-lyase (CYP17). GATA6, SF1, NGFIB, and DAX1 immunoreactivities were all detected in the nuclei of cortical cells of the RSZ. 

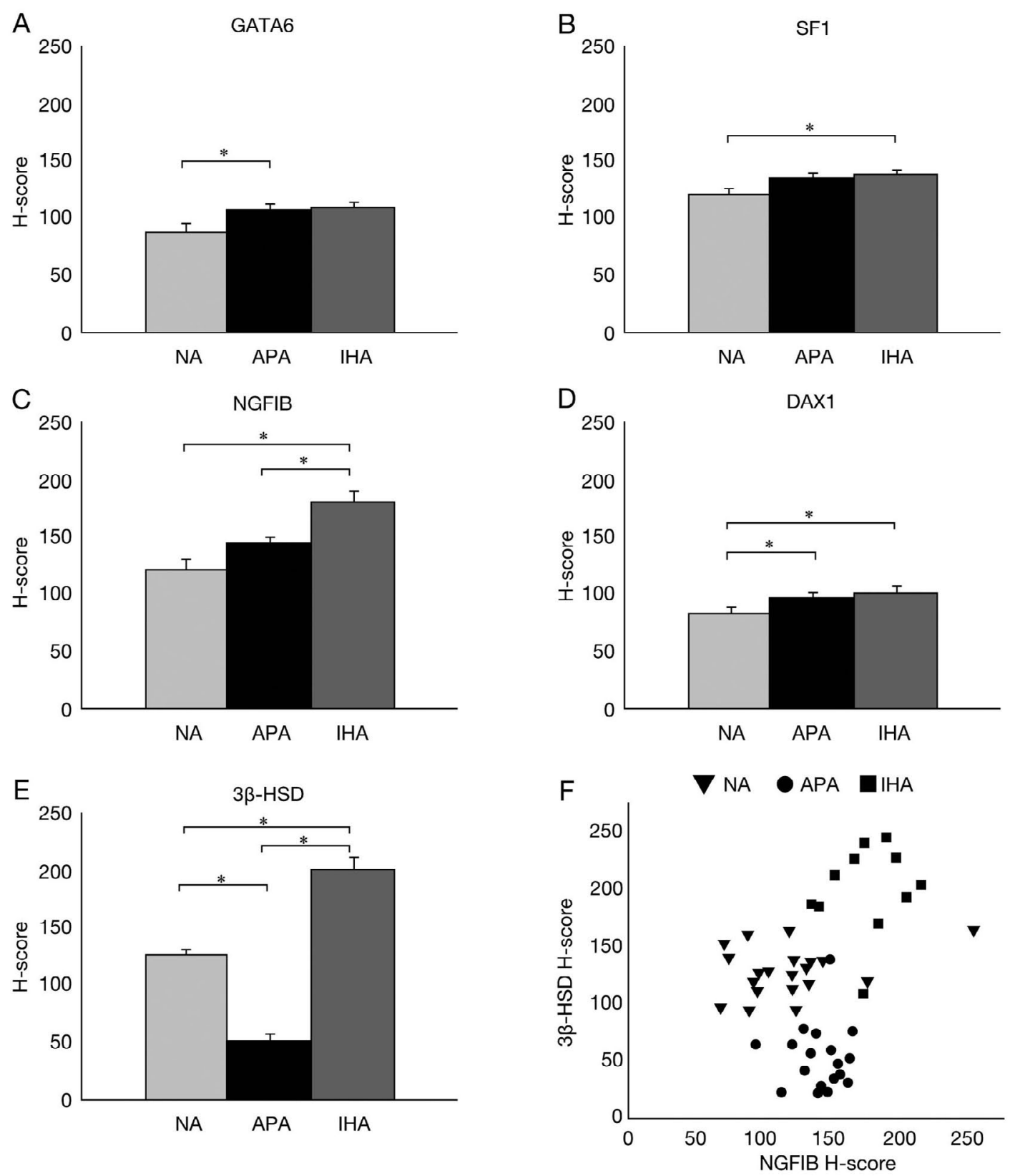

Fig. 4 The relative expression levels (H-score) of GATA6 (A), SF1 (B), NGFIB (C), DAX1 (D) and 3ß-HSD (E) in the ZG of normal adrenal gland (NA) and the remodeled subcapsular zones of idiopathic hyperaldosteronism (IHA) and adjacent adrenal gland to aldosterone-producing adenoma (APA) $(* P<0.05)$. (F) The difference of correlation between relative expression levels of $3 \beta$ HSD and NGFIB in the ZG of NA and the remodeled subcapsular zones of IHA and adjacent adrenal gland to APA.

IHA. These three nuclear transcription factors were also considered to play roles in the subscapular remodeling processes regardless of aldosterone overproduction. GATA6, SF1 and DAX1 are all known to play pivotal roles in developmental processes [13, 22, 23]. In addition, the control of differentiation and functions by GATA6 and DAX1 were reported to be persistent in mature or developed human tissues [24-27]. For instance, GATA6 down-regulation was reported to be involved in the pathogenesis of certain ovarian carcinomas and intimal hyperplasia associated with arterial injuries through its regulation of GATA6 dependent structural proteins $[24,25]$. In addition, the suppression of cadherin 1 by GATA6 up-regulation was 
reported to be associated with bladder smooth muscle hypertrophy, suggesting the importance of GATA6 as a regulator of cellular structure and differentiation [26]. For DAX1, its inactivating mutations have been reported to be associated with X-linked adrenal congenital hypoplasia with adrenal insufficiency, a disease associated with underdevelopment of the adrenal cortex [27]. This finding is suggested, at least in part, to be attributed to DAX1 mediated regulation of adrenal progenitor cells [28]. However, the detailed mechanisms of the involvement of these two factors, which regulated the steroidogenesis in a negative manner, in the development of remodelling of subcapsular areas should be clarified by further investigations.

In contrast to GATA6 and its associated signaling molecules, NGFIB was significantly increased in the remodeled subcapsular zones of IHA compared to APA in addition to being increased in both APA and IHA as compared to normal adrenal. In addition, $3 \beta$-HSD immunoreactivity was significantly higher in the remodeled capsular zone of IHA than those of APA cases and the ZG of NA, while it was significantly lower in APA cases than the ZG of NA. This increased NGFIB in the subcapsular zones of IHA could suggest the following two hypotheses. First, up-regulation of NGFIB in subcapsular zones of IHA compared to APA could account for increased aldosterone production in these cells in IHA, because of the reported correlation between NGFIB and HSD3B2 expression [10]. Second, the increase in NGFIB in the remodeled zones of APA as compared to normal indicated that this trancscription factor also plays a role in the morphological remodeling process in both APA and IHA, regardless of aldosterone overproduction or the staus of steoridogenesis. The potential of this transcription factor to control tissue remodeling has not been previously examined in the adrenal gland but it has been shown to play a role in the lung regeneration process in the early period following pneumonectomy, suggesting its potential as a regulator of tissue remodeling [29].

In conclusion, among the four transcription factors we examined in this study, the relative level of NGFIB expression in the remodeled subcapsular zones of PA cases was the only pivotal marker accounting for the differential expression of steroid enzymes between in the remodeled subcapsular zones of APA and IHA. However, the relative contribution of each factor in the remodeled subcapsular zones should be explored by further studies.

\section{Acknowledgement}

This work was partly supported by the Takeda Science Foundation.

\section{Conflict of Interest}

None of the authors have any potential conflicts of interest associated with this research.

\section{References}

1. Sasano H, Nakamura Y, Moriya T, Suzuki T (2009) Adrenal cortex. Endocrine Pathology-Differential Diagnosis and Molecular Advances (2nd). Springer, New York; 211-226.

2. Nakamura Y, Satoh F, Morimoto R, Kudo M, Takase $\mathrm{K}$, et al. (2011) 18-oxocortisol measurement in adrenal vein sampling as a biomarker for subclassifying primary aldosteronism. J Clin Endocrinol Metab 96: E1272-1278.

3. Boulkroun S, Samson-Couterie B, Dzib JF, Lefebvre H, Louiset E, et al. (2010) Adrenal cortex remodeling and functional zona glomerulosa hyperplasia in primary aldosteronism. Hypertension 56:885-892.

4. Nishimoto K, Nakagawa K, Li D, Kosaka T, Oya M, et al. (2010) Adrenocortical zonation in humans under normal and pathological conditions. J Clin Endocrinol Metab 95:2296-2305.
5. Martin LJ, Tremblay JJ (2005) The human 3beta-hydroxysteroid dehydrogenase/Delta5-Delta4 isomerase type 2 promoter is a novel target for the immediate early orphan nuclear receptor Nur77 in steroidogenic cells. Endocrinology 146:861-869.

6. Jimenez P, Saner K, Mayhew B, Rainey WE (2003) GATA-6 is expressed in the human adrenal and regulates transcription of genes required for adrenal androgen biosynthesis. Endocrinology 144: 4285-4288.

7. Liu Z, Simpson ER (1997) Steroidogenic factor 1 (SF-1) and SP1 are required for regulation of bovine CYP11Agene expression in bovine luteal cells and adrenal Y1 cells. Mol Endocrinol 11: 127-137.

8. Mamluka R, Grebera Y, Meidan R (1999) Hormonal regulation of messenger ribonucleic acid expression for steroidogenic factor-1, steroidogenic acute regulatory protein, and cytochrome P450 side-chain cleavage in 
bovine luteal cells. Biol Reprod 60: 628-634.

9. Shibata H, Kurihara I, Kobayashi S, Yokota K, Suda $\mathrm{N}$, et al. (2003) Regulation of differential COUP-TFcoregulator interactions in adrenal cortical steroidogenesis. J Steroid Biochem Mol Biol 85: 449-456.

10. Bassett MH, Suzuki T, Sasano H, De Vries CJ, Jimenez PT, et al. (2004) The orphan nuclear receptor NGFIB regulates transcription of 3beta-hydroxysteroid dehydrogenase. implications for the control of adrenal functional zonation. J Biol Chem 279: 37622-37630.

11. Wilson TE, Mouw AR, Weaver CA, Milbrandt J, Parker KL (1993) The orphan nuclear receptor NGFI-B regulates expression of the gene encoding steroid 21-hydroxylase. Mol Cell Biol 13: 861-868.

12. Bassett MH, Suzuki T, Sasano H, White PC, Rainey WE (2004) The orphan nuclear receptors NURR1 and NGFIB regulate adrenal aldosterone production. $\mathrm{Mol}$ Endocrinol 18: 279-290.

13. Kiiveri S, Liu J, Westerholm-Ormio M, Narita N, Wilson DB, Voutilainen R, et al. (2002) Differential expression of GATA-4 and GATA-6 in fetal and adult mouse and human adrenal tissue. Endocrinology 143: 3136-3143.

14. Nakamura Y, Suzuki T, Sasano H (2007) Transcription factor GATA-6 in the human adrenocortex: association with adrenal development and aging. Endocr J 54: 783789.

15. Shibata H, Ikeda Y, Mukai T, Morohashi K, Kurihara I, et al. (2001) Expression profiles of COUP-TF, DAX-1, and SF-1 in the human adrenal gland and adrenocortical tumors: possible implications in steroidogenesis. $\mathrm{Mol}$ Genet Metab 74: 206-216.

16. Lu L, Suzuki T, Yoshikawa Y, Murakami O, Miki Y, et al. (2004) Nur-related factor 1 and nerve growth factorinduced clone B in human adrenal cortex and its disorders. J Clin Endocrinol Metab 89: 4113-4118.

17. Sasano H, Shizawa S, Suzuki T, Takayama K, Fukaya T, et al. (1995) Ad4BP in the human adrenal cortex and its disorders. J Clin Endocrinol Metab 80: 2378-2380.

18. Sato Y, Suzuki T, Hidaka K, Sato H, Ito K, et al. (2003) Immunolocalization of nuclear transcription factors, DAX-1 and COUP-TF II, in the normal human ovary: correlation with adrenal 4 binding protein/steroidogenic factor-1 immunolocalization during the menstrual cycle. $J$ Clin Endocrinol Metab 88: 3415-3420.

19. Suzuki T, Sasano H, Takeyama J, Kaneko C, Freije WA, Carr BR, Rainey WE (2000) Developmental changes in steroidogenic enzymes in human postnatal adrenal cortex: immunohistochemical studies. Clin Endocrinol
(Oxf) 53: 739-747.

20. Sakuma I, Suematsu S, Matsuzawa Y, Saito J, Omura M, Maekawa T, Nakamura Y, Sasano H, Nishikawa $\mathrm{T}$ (2013) Characterization of steroidogenic enzyme expression in aldosterone-producing adenoma: a comparison with various human adrenal tumors. Endocr $J$ 60: 329-336.

21. McCarty KS Jr., Miller LS, Cox EB, Konrath J, McCarty KS Sr (1985) Estrogen receptor analyses. Correlation of biochemical and immunohistochemical methods using monoclonal antireceptor antibodies. Arch Pathol Lab Med 109: 716-721.

22. Jin Z, Liu L, Bian W, Chen Y, Xu G, et al. (2009) Different transcription factors regulate nestin gene expression during P19 cell neural differentiation and central nervous system development. J Biol Chem 284: 8160-8173.

23. Niakan KK, Davis EC, Clipsham RC, Jiang M, Dehart $\mathrm{DB}$, et al. (2006) Novel role for the orphan nuclear receptor Dax1 in embryogenesis, different from steroidogenesis. Mol Genet Metab 88:261-271.

24. Cai KQ, Caslini C, Capo-chichi CD, Slater C, Smith ER, et al. (2009) Loss of GATA4 and GATA6 expression specifies ovarian cancer histological subtypes and precedes neoplastic transformation of ovarian surface epithelia. PLoS One 4: e6454.

25. Mano T, Luo Z, Malendowicz SL, Evans T, Walsh K (1999) Reversal of GATA-6 downregulation promotes smooth muscle differentiation and inhibits intimal hyperplasia in balloon-injured rat carotid artery. Circ Res 84: 647-654.

26. Boopathi E, Gomes CM, Goldfarb R, John M, Srinivasan VG, et al. (2011) Transcriptional repression of Caveolin-1 (CAV1) gene expression by GATA-6 in bladder smooth muscle hypertrophy in mice and human beings. Am J Pathol 178: 2236-2251.

27. Choi JH, Park JY, Kim GH, Jin HY, Lee BH, et al. (2011) Functional effects of DAX-1 mutations identified in patients with X-linked adrenal hypoplasia congenita. Metabolism 60: 1545-1550.

28. Wood MA, Hammer GD (2011) Adrenocortical stem and progenitor cells: unifying model of two proposed origins. Mol Cell Endocrinol 336: 206-212.

29. Landesberg LJ, Ramalingam R, Lee K, Rosengart TK, Crystal RG (2001) Upregulation of transcription factors in lung in the early phase of postpneumonectomy lung growth. Am J Physiol Lung Cell Mol Physiol 281: L1138-1149. 